\title{
A self-sustaining ultrahigh-frequency nanoelectromechanical oscillator
}

\author{
X. L. FENG ${ }^{1}, 2$, C. J. WHITE2 2 A. HAJIMIRI2 AND M. L. ROUKES ${ }^{1 *}$ \\ ${ }^{1}$ Kavli Nanoscience Institute, MC 114-36, California Institute of Technology, Pasadena, California 91125, USA \\ ${ }^{2}$ Electrical Engineering, MC 136-93, California Institute of Technology, Pasadena, California 91125, USA \\ *e-mail: roukes@caltech.edu
}

Sensors based on nanoelectromechanical systems vibrating at high and ultrahigh frequencies ${ }^{1}$ are capable of levels of performance that surpass those of larger sensors. Nanoelectromechanical devices have achieved unprecedented sensitivity in the detection of displacement ${ }^{2}$, mass ${ }^{3}$, force ${ }^{4}$ and charge ${ }^{5}$. To date, these milestones have been achieved with passive devices that require external periodic or impulsive stimuli to excite them into resonance. Here, we demonstrate an autonomous and self-sustaining nanoelectromechanical oscillator that generates continuous ultrahigh-frequency signals when powered by a steady d.c. source. The frequencydetermining element in the oscillator is a $428 \mathrm{MHz}$ nanoelectromechanical resonator that is embedded within a tunable electrical feedback network to generate active and stable self-oscillation. Our prototype nanoelectromechanical oscillator exhibits excellent frequency stability, linewidth narrowing and low phase noise performance. Such ultrahighfrequency oscillators provide a comparatively simple means for implementing a wide variety of practical sensing applications. They also offer intriguing opportunities for nanomechanical frequency control, timing and synchronization.

Active oscillators spontaneously generate self-sustaining periodic signals by extracting power from steady (d.c.) sources. This distinguishes them from passive resonators-which are characterized, in contrast, by a damped response to impulsive stimuli-and makes them invaluable for applications in precision timekeeping $^{6}$, communications ${ }^{7}$ and sensing ${ }^{8}$, which require continuous a.c. signals. Oscillators based upon the mechanical vibrations of crystals such as quartz resonators have long been ubiquitous in electronics, as a result of their simplicity and excellent stability for frequency control applications ${ }^{9}$. Over the past few decades, there has been considerable incentive to miniaturize such mechanical resonators, in order to integrate them on-chip with electronic components to add frequencyselection and tuning elements ${ }^{10,11}$. In particular, it is desirable to realize highly accurate and stable clocks or frequency references with integrated, chip-based systems using miniaturized acoustically resonant devices.

Resonant nanoelectromechanical systems (NEMS) have recently generated significant interest in this area because of their ultrahigh operating frequencies ${ }^{1}$, small size, very low operating power and high quality factors $(Q)$. In fact, the values of $Q$ achieved by NEMS typically greatly exceed what can be obtained using electronic components. In parallel with the quest for miniaturized frequency references, which motivates the development of nanoscale resonators, efforts are particularly focused on exploiting their unprecedented responsivity for a variety of sensing applications in science and technology ${ }^{2-5}$. Accordingly, there is significant impetus for developing self-sustaining NEMS oscillators for ultrasensitive, frequency-shift-based sensing. However, for reasons we describe below, ultrahigh-frequency (UHF; $\geq 300 \mathrm{MHz}$ ) signal generation based on nanoscale devices has remained elusive, despite recent advances in the development of high-frequency, microelectromechanical systems (MEMS) oscillators ${ }^{12,13}$. Here, for the first time, we harness the fundamental-mode vibrations of a nanoscale mechanical device to obtain stable, self-sustaining oscillations at UHF-at fundamental frequencies significantly higher than typically achieved with conventional quartz crystal oscillators $(\sim 10 \mathrm{MHz})$ or with the recently developed MEMS oscillators (up to $\sim 60 \mathrm{MHz})^{13}$.

Our self-sustaining NEMS oscillator, as shown in Fig. 1a, consists of an electrical feedback circuit with an embedded UHF NEMS resonator as the frequency-determining element. This element's motion is transduced into an electrical signal, amplified, and then fed back (with adjustable gain and phase) to the NEMS resonator. Stable mechanical vibration of the NEMS element is maintained with d.c. power input to the amplifier in this feedback loop. The elusiveness of successfully producing UHF and microwave-frequency oscillators based on NEMS stems from the difficulty in obtaining optimal transduction of their miniscule displacements. After transduction into the electrical domain, the motion-induced signals generated by such scaled-down resonators are extremely small, making them exceptionally difficult to tune and control in an electrical feedback circuit. Specifically, the unavoidable parasitic coupling between a NEMS device and its macroscale surroundings generally overwhelms its intrinsic electromechanical response. We overcome this with a precisely tunable detection circuit (see Supplementary Information) that deeply nulls the unavoidable parasitics, to allow transduction of UHF NEMS vibratory motions into electrical signals with high efficiency. For a doubly clamped silicon carbide $(\mathrm{SiC})$ beam with dimensions $1.65 \mu \mathrm{m}(L) \times 120 \mathrm{~nm}(w) \times 80 \mathrm{~nm}(t)$, and a fundamental flexural mode resonance of $\omega_{0} / 2 \pi=428.2 \mathrm{MHz}$ and $Q \sim 2,500$, this enables us to achieve a resonant, open-loop electrical response that is $8 \mathrm{~dB}$ above the background. (See Methods and Supplementary Information for details about device fabrication, resonator displacement transduction, electronic detection circuitry and measurements.) 
a
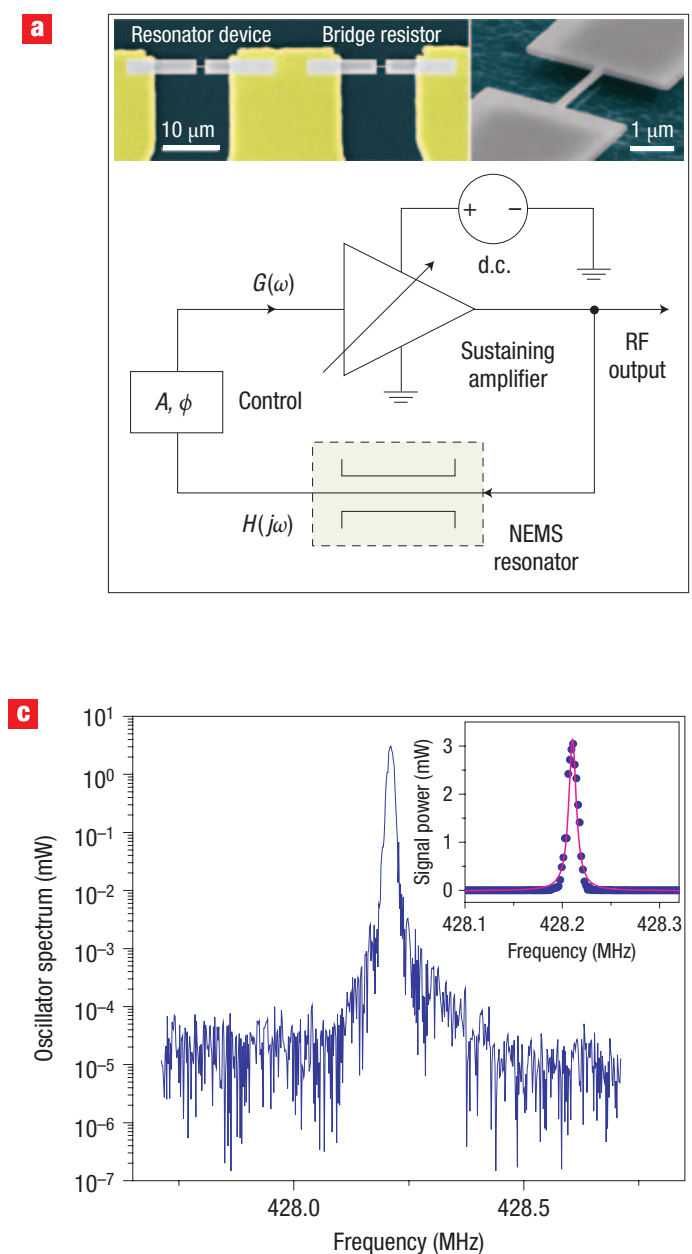
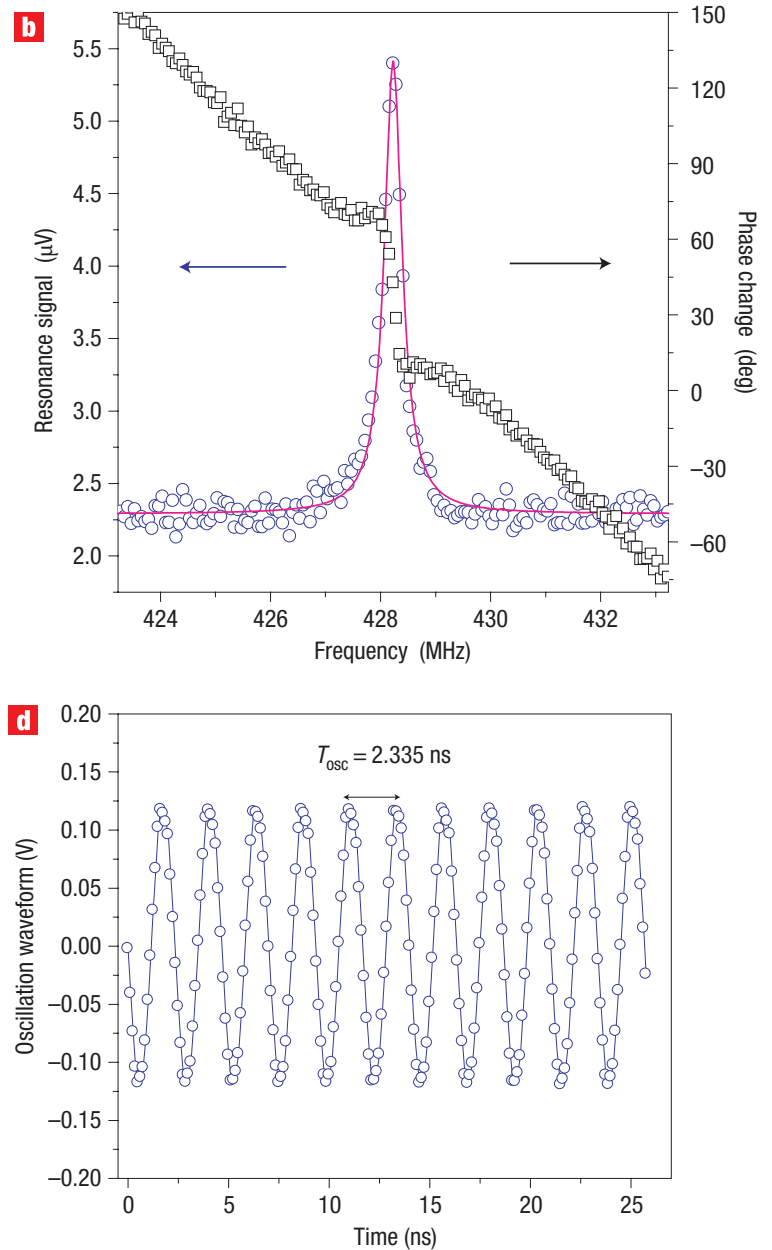

Figure 1 Self-sustaining UHF NEMS oscillator. a, Simplified circuit schematic (see Supplementary Information, Fig. S1, for more detail) for the self-sustaining oscillator, which includes the frequency-determining UHF NEMS resonator and the tunable electronic feedback loop. The inset shows scanning electron micrographs depicting the device (right) and its embedding electrical-bridge configuration (left), from which a large resonant response is obtained by efficiently nulling parasitic signals. b, Open-loop electrical-domain amplitude (blue open circles) and phase (black open squares) signals from the $428 \mathrm{MHz}$ NEMS resonator (referred to the input of the preamplifier). A large coherent response $8 \mathrm{~dB}$ above the background is observed. The magenta line is a fit of the signal amplitude to the model for a damped driven harmonic resonator. c, Output power spectrum of the NEMS oscillator (on a logarithmic scale) as a function of frequency measured with a $100 \mathrm{kHz}$ resolution bandwidth. The inset shows the output power spectrum on a linear scale: the linewidth narrowing can be clearly seen (as compared with Fig. 1b). d, The clean, stable, sinusoidal time-domain oscillation waveform of the closed-loop NEMS oscillator measured by a high-speed digital oscilloscope.

To realize a NEMS-based oscillator, it is critical to obtain a clean resonant response well above the background over a wide frequency range (Fig. 1b). Under such conditions, it becomes possible to calibrate and adjust the open-loop gain and phase changes to satisfy the Barkhausen criterion ${ }^{7}, \quad G\left(\omega_{0}\right) H\left(j \omega_{0}\right)=1$, on resonance, $\omega=\omega_{0}$. Here, $G(\omega)$ is the gain of the feedback loop and $H(j \omega)$ the transfer function of the frequency-determining NEMS element; we represent both as complex functions. Fulfilling the Barkhausen condition ensures that the electronic feedback exactly compensates the nanoresonator's intrinsic and extrinsically coupled damping, thereby sustaining continuous oscillation. This requires precise tuning of the loop gain and phase. In the steady state, the amplitude of oscillation is stabilized by nonlinearity in the electronic gain, $G(\omega)$, at large signal amplitudes. The steady-state self-oscillations of the system are readily characterized by the frequency-domain power spectrum of the oscillator output (Fig. 1c). Figure 1d demonstrates the clean, stable, time-domain oscillation waveforms from the output of the closed-loop NEMS oscillator, displaying a period of $T_{\mathrm{osc}}=2.335 \mathrm{~ns}$.

A compelling feature of the NEMS oscillator is the linewidth narrowing ${ }^{14}$ in its frequency-domain spectrum when compared with that of the passive resonator. The driven resonator's response has a lorentzian line broadening (Fig. 1b) due to its damping (finite $Q$ ), and the linewidth, set by the damping rate, is $\Delta_{\text {res }}=\omega_{0} /\left(2 \pi Q_{\text {res }}\right) \approx 0.1713 \mathrm{MHz}$. Following the same convention of full-width at half-maximum (FWHM) of the power signal, the linewidth of the NEMS oscillator is measured to be $\Delta_{\text {osc }} \equiv \mathrm{FWHM} \approx 9 \mathrm{kHz}$. The linewidth narrowing ratio is $\Delta_{\text {osc }} / \Delta_{\text {res }} \approx 1 / 19$, which corresponds to an effective $Q$ factor of $Q_{\text {osc,eff }}=\omega_{0} /\left(2 \pi \Delta_{\text {osc }}\right) \approx 47,580$ for the oscillator. The inset of Fig. 1c approximately illustrates the oscillator linewidth narrowing with a brute-force lorentzian fit to the oscillator power signal. Note here that the oscillator spectrum is strictly lorentzian 


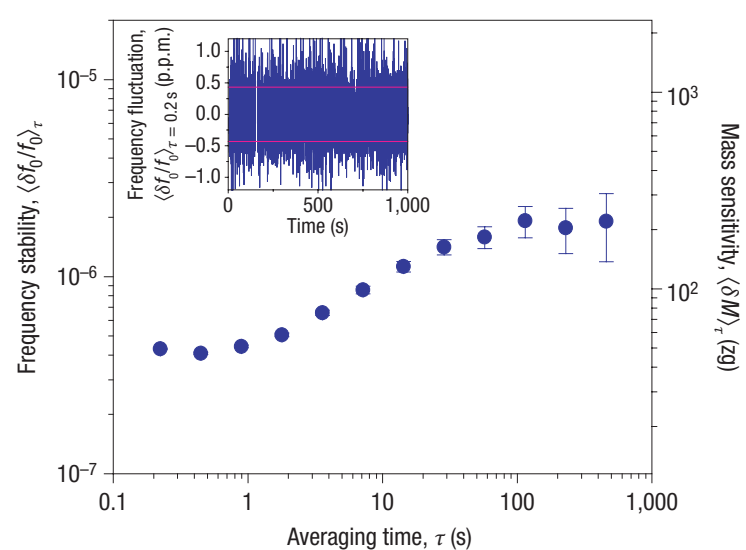

Figure 2 Frequency stability and mass sensitivity of the self-sustaining UHF NEMS oscillator. Measured frequency stability $\left\langle\delta f_{0} / f_{0}\right\rangle_{\tau}$ (on a logarithmic scale) as a function of averaging time $\tau$. The inset shows the 'instantaneous' fractional frequency fluctuation (on a linear scale in units of parts per million of $\sim 4 \times 10^{-7}$ for $\tau=0.2 \mathrm{~s}$. This stability level is achieved for short-term averaging, for example, $\tau=0.2-1 \mathrm{~s}$. This plot also shows the UHF NEMS oscillator's corresponding mass sensitivity, $\langle\delta M\rangle_{\tau} \approx 2 M_{\text {eff }}\left\langle\delta f_{0} / f_{0}\right\rangle_{\tau}$, again as a function of averaging time $\tau$. Given the NEMS device's active mass of $M_{\text {eff }}=57.8 \mathrm{fg}$, the system achieves a short-term mass resolution of $\sim 50 \mathrm{zg}$.

when the system noise is white. In this case the oscillator phase noise is a diffusive process, which can be equivalently viewed as a virtual damping effect $^{14}$ causing a finite spectral linewidth, similar to the resonator case. When the noise spectral density is not white, the oscillator power signal deviates from lorentzian and such a fit is only an approximation. Nonetheless, one can still estimate $\Delta_{\text {osc }}$ and $Q_{\text {osc,eff }}$ by using the FWHM.

The frequency stability of our prototype NEMS oscillator is particularly noteworthy. For frequency-shift sensing applications, frequency-fluctuation noise imposes an ultimate limit to detection sensitivity. We characterize the NEMS oscillator's frequency stability by its fractional frequency fluctuations, $\left\langle\delta f_{0} / f_{0}\right\rangle_{\tau}$, which are a function of averaging time $\tau$, $\left\langle\delta f_{0} / f_{0}\right\rangle_{\tau}=\left[(1 /(N-1)) \cdot \sum_{i=1}^{N}\left(\left(\bar{f}_{i+1}-\bar{f}_{i}\right) / f_{0}\right)^{2}\right]^{1 / 2}$, where $\bar{f}_{i}$ is the averaged frequency in the $i$ th discrete time interval of $\tau$. As shown in Fig. 2, we have achieved $\left\langle\delta f_{0} / f_{0}\right\rangle_{\tau} \approx 4 \times 10^{-7}$ for short times $(\tau<1 \mathrm{~s})$ and $\sim 1 \times 10^{-6}$ for longer times $(\tau>100 \mathrm{~s})$. Such frequency stability is particularly promising for mass sensing: recent advances with ultraminiature mechanical vibratory sensors have yielded unprecedented zeptogram-scale mass sensitivity for applications in vacuum ${ }^{3}$ and at atmospheric pressure $^{15}$, and attogram sensitivity for fluidic-based sensing ${ }^{16}$. The NEMS oscillator described here-with an active resonator mass $M_{\text {eff }}$ of only $57.8 \mathrm{fg}$ and high mass responsivity ${ }^{17}$ of $|\mathfrak{R}|=\omega_{0} /\left(2 M_{\text {eff }}\right)=3.7 \mathrm{~Hz} \mathrm{zg}^{-1}$-permits a mass resolution $\langle\delta M\rangle_{\tau} \approx 2 M_{\text {eff }}\left\langle\delta f_{0} / f_{0}\right\rangle_{\tau}$ at the $\sim 50 \mathrm{zg}$ level with short averaging times, $\tau \sim 1-1,000 \mathrm{~ms}$. This is a trillion-fold improvement over typical commercial quartz crystal microbalances ${ }^{8,18}$. The prototype NEMS oscillator is also readily applicable for ultrasensitive force detections in real time $e^{4}$. The present $428 \mathrm{MHz}$ doubly clamped beam device has a relatively large stiffness of $k_{\text {eff }}=418 \mathrm{~N} \mathrm{~m}^{-1}$ and a fundamental force sensitivity of $S_{\mathrm{F}}^{1 / 2}=\left(4 k_{\mathrm{B}} T M_{\mathrm{eff}} \omega_{0} / Q\right)^{1 / 2} \approx 275 \mathrm{aN} \mathrm{Hz} z^{-1 / 2}$, limited by its thermomechanical motion. Here $k_{\mathrm{B}}$ is the Boltzmann constant and $T$ the temperature. Combined with cantilever devices of high $Q$ values and lower force constants specifically designed for force

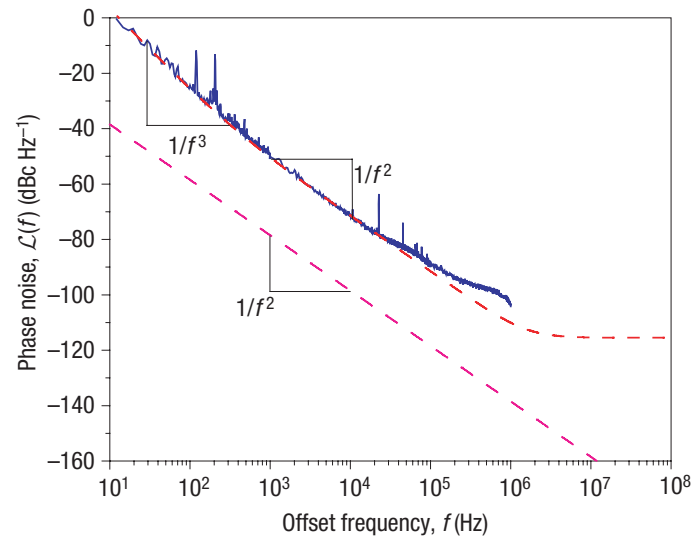

Figure 3 Phase noise performance of the self-sustaining UHF NEMS oscillator. The blue solid line is the measured phase noise data, demonstrating a $1 / f^{2}$ power law and thus thermal-noise-limited performance in most of the frequency range of interest. A Leeson-type empirical fit (red dashed line) further demonstrates that, close to the carrier for offset frequency $f<300 \mathrm{~Hz}$, the phase noise approximately follows a $1 / f^{3}$ power law as flicker noise arises in this range. The carrier signal power is $P_{\mathrm{C}} \approx 0.32 \mathrm{nW}$. The achieved phase noise performance is presently limited by extrinsic electronic noise from the preamplifier and the NEMS transducer. The ultimate phase noise (magenta dashed line) is limited by thermomechanical fluctuations in the NEMS device, which could be approached by further engineering of the transduction and electronic detection.

sensing ${ }^{4,19}$, NEMS oscillators can attain much higher force sensitivities (in the low- and sub-attonewton regimes) while providing wide bandwidth operation ${ }^{20}$.

Understanding and minimizing oscillator phase noise is key to attaining high precision and stability. Phase noise is traditionally quantified as the sideband power spectral density at an offset frequency $f$, normalized by the carrier signal power $P_{\mathrm{C}}$ (the power sustains the resonator's motion). This is written as $\mathcal{L}(f)=10 \log \left[P_{\text {sideband }}\left(f_{\mathrm{C}}+f\right) / P_{\mathrm{C}}\right]$, and has units of $\mathrm{dBc} \mathrm{Hz}{ }^{-1}$ (decibels below the carrier per hertz) ${ }^{7,14,21}$. As shown in Fig. 3, the measured phase noise spectrum of the prototype UHF NEMS oscillator closely follows a $1 / f^{2}$ power law in the $300 \mathrm{~Hz}$ to $1 \mathrm{MHz}$ offset frequency range. This indicates that phase noise in this regime originates predominantly from fluctuating sources with white spectra ${ }^{14,21}$. In the present case, these are largely the electronic thermal noise from the NEMS displacement transducer and its readout amplifier. Close in to the resonance peak frequency (carrier), we observe phase noise exhibiting $1 / f^{3}$ behaviour, which is consistent with $1 / f$ (flicker) noise mechanisms expected in this regime. As Fig. 3 displays, we find that an empirical fit to a Leeson-type model ${ }^{21,22}$ - that is, $\mathcal{L}(f)=10 \log \left[\left(2 F_{n} k_{\mathrm{B}} T / P_{\mathrm{C}}\right) \cdot\left(1+\left(f_{0} / 2 Q f\right)\right)^{2}\left(1+\left(f_{1 / f^{3}} / f\right)\right)\right]$ agrees very well with the measured phase noise data. This fit yields an equivalent noise factor of $F_{n}=1.4$ and corner (knee) offset frequency for $1 / f^{3}$ phase noise (that is, $1 / f$ frequency noise) of $f_{1 / f^{3}} \approx 300 \mathrm{~Hz}$. The time-domain oscillation stability and frequency-domain phase noise are correlated, as can be seen in Figs 2 and 3. Whereas white and flicker noise affect the short term performance, it is drift and aging that compromise the oscillator's long-term stability. From measurements and analyses of the oscillator's phase fluctuations (see Methods and Supplementary Information), we deduce that the combination of electrical-domain thermal noise in the transducer and loop amplifier dominates the mechanical-domain noise of the NEMS 
resonator itself in this oscillator realization. Note that, for communications applications, the phase noise performance of the prototype NEMS oscillator needs further engineering (for example, in improving the device carrier power $P_{\mathrm{C}}$ ). MEMS oscillators based upon micromachined devices operating in much stiffer (for example, bulk acoustic) modes ${ }^{13,23}$, although not optimal for sensing applications, are now approaching the phase noise specifications of macroscale quartz crystal oscillators $^{24}$. Further miniaturization of film-bulk acoustic resonators (FBARs) ${ }^{23}$ and devices using other stiff modes, and arraying ensembles of devices, should significantly enhance the power handling and phase noise performance of NEMS oscillators.

The ultimate limits of phase noise performance are imposed by the NEMS resonator's intrinsic thermomechanical fluctuations ${ }^{25}$. The corresponding phase noise in this limit is $\mathcal{L}(f)=10 \log$ $\left[\left(k_{\mathrm{B}} T / 2 P_{\mathrm{C}} Q^{2}\right) \cdot\left(f_{0} / f\right)^{2}\right]$, which is also displayed in Fig. 3. We can also represent this limit by its corresponding fractional frequency fluctuations, again as a function of averaging time $\tau$, $\left\langle\delta f_{0} / f_{0}\right\rangle_{\tau}=(1 / Q) \cdot\left(\pi k_{\mathrm{B}} T / P_{\mathrm{C}} \tau\right)^{1 / 2}$. For the NEMS device in this work, this yields an ultimate limit of $\left\langle\delta f_{0} / f_{0}\right\rangle_{\tau} \approx 5 \times 10^{-10}$ for $\tau \sim 1 \mathrm{~s}$. Hence, with further optimization, up to a thousand-fold improvement in performance may be possible over our present extrinsic-noise-limited value, $\left\langle\delta f_{0} / f_{0}\right\rangle_{\tau} \approx 4 \times 10^{-7}$ for $\tau=1 \mathrm{~s}$.

Self-sustaining NEMS oscillators enable a wide spectrum of applications. They offer significant advantages over previously used methods of frequency-shift detection (for example, phaselocked loops ${ }^{3,15}$ ) as no source of external excitation is required. This yields an immense simplification of design that is crucial for next-generation, highly multiplexed sensing applications involving large arrays of devices. Additionally, NEMS oscillators will enable important applications in metrology at the nanoscale, providing a generic approach to wideband and real-time transduction of fundamental physical processes ${ }^{26}$. Furthermore, large arrays of coupled tunable mechanical oscillators may offer promising prospects for nanomechanical timing, signal processing 27 and noise suppression through oscillator synchronization $^{28}$. It should be noted that synchronization requires the coupling of self-sustaining, autonomous oscillators, rather than passive resonators ${ }^{29}$. Arrays of NEMS oscillators may also provide an intriguing means of simulating biological systems, such as pacemaker cells and neural oscillators ${ }^{30}$, where large numbers of oscillators spontaneously synchronize to perform collective functions.

\section{METHODS}

\section{FABRICATION AND DETECTION OF UHF NEMS RESONATORS}

We fabricated the UHF NEMS resonators from an 80-nm single-crystal SiC epilayer grown upon a silicon substrate, using a surface nanomachining process as detailed elsewhere ${ }^{31}$. In brief, for the structures shown in the insets to Fig. 1a, the contacting gold electrodes were defined by photolithography, deposition of a 80 -nm gold layer and a liftoff process. The NEMS devices were successively defined by e-beam lithography, metallized by a thermally evaporated $\sim 5-10-\mathrm{nm}$ layer of titanium on top of $\sim 30-40-\mathrm{nm}$ aluminium, and then a liftoff process. The metallization was engineered so that the device's two-terminal resistance was typically $\sim 100 \Omega$ at room temperature and $\sim 50 \Omega$ at low temperatures to facilitate electronic characterization at UHF.

The device's fundamental, in-plane, flexural mode resonance was excited and detected using magnetomotive transduction, which we find is particularly suitable for doubly clamped beam resonators in the UHF range1 ${ }^{1,31}$.

Measurements were carried out in vacuo, with the NEMS resonator regulated at a temperature of $T=22 \mathrm{~K}$ and immersed in an $8 \mathrm{~T}$ magnetic field. Note that although magnetomotive transduction is probably the most successful scheme for UHF nanobeam or nanowire resonators to date that does not require frequency downconversion, it should not be taken as a fundamental or intrinsic ingredient for UHF NEMS. Efficient actuation and wideband sensitive detection are being actively pursued through the adoption of new materials and nanomechanical coupling effects ${ }^{32,33}$ and new circuit techniques, to enable integrated, room-temperature transduction of UHF nanobeams and nanowires.

Here, electronic displacement detection was optimized through the use of a tunable, high-resolution bridge circuit to deeply null the background response arising from parasitic effects, impedance mismatch, and so on, to yield excellent signal-to-background ratios (SBRs) of the order of $\sim 5-10 \mathrm{~dB}$, on resonance (see Supplementary Information, Fig. S1). This is unprecedented in the detection of UHF NEMS resonators and is a significant improvement over the typical $\sim 0.1 \mathrm{~dB}$ (or even smaller) SBRs previously achieved with UHF NEMS devices ${ }^{1,31}$

\section{UHF NEMS DETECTION NOISE AND OSCILLATOR PHASE NOISE}

The electrically transduced NEMS resonator can be represented by its d.c. resistance in series with its electromechanical impedance (see Supplementary Information, Fig. S1). The latter is modelled by a parallel $R_{\mathrm{m}} C_{\mathrm{m}} L_{\mathrm{m}}$ electrical resonator ${ }^{34}$. Here, the subscript $\mathrm{m}$ denotes that these arise from the resonator's motion. Note that the series resonant circuit model for capacitive transduction ${ }^{13}$ widely used in MEMS often involves a very large motional resistance (typically in the $\mathrm{k} \Omega$ to $\mathrm{M} \Omega$ ranges). Here, our NEMS devices are described by a parallel resonant circuit model and are close to $50 \Omega$ in total impedance. This is more suitable for UHF in terms of impedance matching in transduction and choice of sustaining amplifiers. The detection noise floor reached in these experiments is limited by the post-transducer amplifier's voltage noise (with a calibrated noise temperature of $T_{\mathrm{n}}=9 \mathrm{~K}$ ) in combination with Johnson noise of the series d.c. resistance. This combination yields a total voltage noise of $\sim 0.238 \mathrm{nV} \mathrm{Hz}^{-1 / 2}$ (referred to the input of the preamplifier). This translates into an effective displacement sensitivity of $\sim 12.8 \mathrm{fm} \mathrm{Hz}^{-1 / 2}$. At $T=22 \mathrm{~K}$, the ultimate limit to the displacement noise floor for this device is set by thermomechanical fluctuations and corresponds to $\sim 1.64 \mathrm{fm} \mathrm{Hz}^{-1 / 2}$. This thermomechanical motion generates, in the presence of the $8 \mathrm{~T}$ magnetic field, an equivalent electromotive-force voltage noise floor of $\sim 0.0305 \mathrm{nV} \mathrm{Hz}^{-1 / 2}$. The onset of nonlinearity arising from the Duffing instability ${ }^{35}$ for this device is $\sim 1.6 \mathrm{~nm}$, so the device possesses an intrinsic dynamic range ${ }^{35}$ of $114 \mathrm{~dB}$. However, in these experiments the bottom-most $18 \mathrm{~dB}$ of the intrinsic dynamic range is forfeited due to the imperfect noise match between the transducer and the subsequent readout amplifier, as described above. The dynamic range available to the system, including both the NEMS resonator and detection circuitry, is therefore about $96 \mathrm{~dB}$.

For the self-sustaining NEMS oscillator, the total phase noise can be viewed as the sum of several parts. First, fluctuations associated with the vibrationinduced motional resistance, $R_{\mathrm{m}} \sim 1.5 \Omega$, appearing in the parallel $R_{\mathrm{m}} C_{\mathrm{m}} L_{\mathrm{m}}$ representation of the NEMS electromechanical impedance (see Supplementary Information, Fig. S1), correspond to the thermomechanical displacement noise of the resonator transduced into the electrical domain. In this domain, additional electrical noise originates from the d.c. resistance of the transducer, which we model as an equivalent series resistance $R_{\mathrm{S} \text {,eq }}=26.1 \Omega$ Under the conditions of this experiment $R_{\mathrm{S}, \mathrm{eq}}>R_{\mathrm{m}}$, so optimal engineering can further improve the measured noise floor.

Received 14 February 2008; accepted 16 April 2008; published 25 May 2008

References

1. Huang, X. M. H., Zorman, C. A., Mehregany, M. \& Roukes, M. L. Nanodevice motion at microwave frequencies. Nature 421, 496 (2003)

2. LaHaye, M. D., Buu, O., Camarota, B. \& Schwab, K. C. Approaching the quantum limit of a nanomechanical resonator. Science 304, 74-77 (2004).

3. Yang, Y. T., Callegari, C., Feng, X. L., Ekinci, K. L. \& Roukes, M. L. Zeptogram-scale nanomechanical mass sensing. Nano Lett. 6, 583-586 (2006).

4. Rugar, D., Budakian, R., Mamin, H. J. \& Chui, B. W. Single spin detection by magnetic resonance force microscopy. Nature 430, 329-332 (2004).

5. Cleland, A. N. \& Roukes, M. L. A nanometre-scale mechanical electrometer. Nature 392, $160-162$ (1998).

6. Audoin, C. \& Guinot, B. The Measurement of Time: Time, Frequency, and the Atomic Clock (trans. Lyle, S.) (Cambridge Univ. Press, New York, 2001).

7. Hajimiri, A. \& Lee, T. H. The Design of Low Noise Oscillators (Kluwer Academic Publishers, Norwell, 1999).

8. Ward, M. D. \& Buttry, D. A. In situ interfacial mass detection with piezoelectric transducers. Science $249,1000-1007$ (1990).

9. Cady, W. G. The piezo-electric resonator. Proc. IRE 10, 83-114 (1922).

10. Nathanson, H. C., Newell, W. E., Wickstrom, R. A. \& Davis, J. R. Jr. The resonant gate transistor. IEEE Trans. Electron. Dev. ED-14, 117-133 (1967).

11. Newell, W. E. Miniaturization of tuning forks. Science 161, 1320-1326 (1968)

12. Nguyen, C. T. C. \& Howe, R. T. An integrated CMOS micromechanical resonator high-Q oscillator IEEE J. Solid State Circ. 34, 440-455 (1999). 
13. Lin, Y. W. et al. Series-resonant VHF micromechanical resonator reference oscillators. IEEE J. Solid State Circ. 39, 2477-2491 (2004).

14. Ham, D. \& Hajimiri, A. Virtual damping and Einstein relation in oscillators. IEEE J. Solid State Circ. 38, 407-418 (2003)

15. Li, M., Tang, H. X. \& Roukes, M. L. Ultra-sensitive NEMS-based cantilevers for sensing, scanned probe and very high-frequency applications. Nature Nanotech. 2, 114-120 (2007).

16. Burg, T. P. et al. Weighing of biomolecules, single cells and single nanoparticles in fluid. Nature 446, 1066-1069 (2007).

17. Ekinci, K. L., Yang, Y. T. \& Roukes, M. L. Ultimate limits to inertial mass sensing based upon nanoelectromechanical systems. J. Appl. Phys. 95, 2682-2689 (2004).

18. Rodahl, M., Höök, F., Krozer, A., Brzezinski, P. \& Kasemo, B. Quartz crystal microbalance setup for frequency and Q-factor measurements in gaseous and liquid environments. Rev. Sci. Instrum. 66, 3924-3930 (1995).

19. Arlett, J. L., Maloney, J. R., Gudlewski, B., Muluneh, M. \& Roukes, M. L. Self-sensing micro- and nanocantilevers with attonewton-scale force resolution. Nano Lett. 6, 1000-1006 (2006).

20. Albrecht, T. R., Grütter, P., Horne, D. \& Rugar, D. Frequency modulation detection using high-Q cantilevers for enhanced force microscope sensitivity. J. Appl. Phys. 69, 668-673 (1991).

21. Lee, T. H. \& Hajimiri, A. Oscillator phase noise: a tutorial. IEEE J. Solid State Circ. 35, $326-336$ (2000).

22. Leeson, D. B. A simple model of feedback oscillator noise spectrum. Proc. IEEE 54, 329-330 (1966).

23. Otis, B. P. \& Rabaey, J. M. A $300 \mu \mathrm{W} 1.9 \mathrm{GHz}$ CMOS oscillator utilizing micromachined resonators. IEEE J. Solid State Circ. 38, 1271-1274 (2003).

24. Vig, J. R. \& Kim, Y. Noise in microelectromechanical system resonators.

IEEE Trans. Ultrason. Ferroelectr. Freq. Contr. 46, 1558-1565 (1999).

25. Cleland, A. N. \& Roukes, M. L. Noise processes in nanomechanical resonators. J. Appl. Phys. 92 $2758-2769$ (2002).

26. Schwab, K. C. \& Roukes, M. L. Putting mechanics into quantum mechanics. Phys. Today 58, 36-42 (July 2005).

27. Nguyen, C. T. C., Katehi, L. P. B. \& Rebeiz, G. M. Micromachined devices for wireless communications. Proc. IEEE 86, 1756-1768 (1998).
28. Cross, M. C., Zumdieck, A., Lifshitz, R. \& Rogers, J. L.

Synchronization by nonlinear frequency pulling. Phys. Rev. Lett. 93, 224101 (2004).

29. Pikovsky, A., Rosenblum, M. \& Kurths, J. Synchronization: A Universal Concept in Nonlinear Sciences (Cambridge Univ. Press, 2001).

30. Varela, F., Lachaux, J. P., Rodriguez, E. \& Martinerie, J. The brainweb: phase synchronization and large-scale integration. Nature Rev. Neurosci. 2, 229-239 (2001).

31. Huang, X. M. H., Feng, X. L., Zorman, C. A., Mehregany, M. \& Roukes, M. L. VHF, UHF and microwave frequency nanomechanical resonators. New J. Phys. 7, 247 (2005).

32. Lin, Y. W., Li, S. S., Xie, Y., Ren, Z. \& Nguyen, C. T. C. Vibrating micromechanical resonators with solid dielectric capacitive transducer gaps, in Proc. IEEE Int. Freq. Contr. Symp., August 29-31, 128-134 (IEEE, Vancouver, Canada, 2005).

33. Masmanidis, S. C. et al. Multifunctional nanomechanical systems via tunably coupled piezoelectric actuation. Science 317, 780-783 (2007).

34. Cleland, A. N. \& Roukes, M. L. External control of dissipation in a nanometer-scale radiofrequency mechanical resonator. Sens. Actuators A 72, 256-261 (1999).

35. Postma, H. W. C., Kozinsky, I., Husain, A. \& Roukes, M. L. Dynamic range of nanotube- and nanowire-based electromechanical systems. Appl. Phys. Lett. 86, 223105 (2005)

Supplementary Information accompanies this paper at www.nature.com/naturenanotechnology.

\section{Acknowledgements}

We thank C.T.C. Nguyen, J.R. Vig, M.C. Cross and R. Lifshitz for helpful discussions. We thank M. Mehregany and C.A. Zorman for providing $\mathrm{SiC}$ material. We acknowledge support from DARPA/SPAWAR under grant N66001-02-1-8914.

\section{Author information}

Reprints and permission information is available online at http://npg.nature.com/reprintsandpermissions/. Correspondence and requests for materials should be addressed to M.L.R. 